International Journal of Engineering \& Technology, $7(3.29)(2018) 232-235$
SPC
Website $: \frac{w w \text {. sciencepubco.com/index.php/IJET }}{\text { Research paper }}$

\title{
CFD analysis of convergent-divergent nozzle flow and base pressure control using micro-JETS
}

\author{
Ambareen Khan ${ }^{1}$, Abdul Aabid ${ }^{2}$, S. A. Khan ${ }^{2}$ \\ ${ }^{1}$ School of Aerospace Engineering, University Sains Malaysia, Nibong Tebal, Penang, Malaysia \\ ${ }^{2}$ Department of Mechanical Engineering, International Islamic University Malaysia, Kuala Lumpur, Malaysia \\ *Corresponding author E-mail: sakhan@iium.edu.my
}

\begin{abstract}
This paper presents numerical study was undertaken to identify the use of the micro-jets to regulate the pressure in the region from twodimensional convergent-divergent (CD) Nozzle. At the exit of the divergent nozzle in the base region $1 \mathrm{~mm}$ of two micro-jets orifice diameter has been arranged at ninety degrees at pcd $13 \mathrm{~mm}$ to control base pressure. The inertia level at the inlet to suddenly expanded duct was 1.87 . The micro-jets are suddenly expanded into a two-dimensional planar area ratio of 3.24 . The $\mathrm{L} / \mathrm{D}$ of the duct was $1,2,4,5$, 6,8 and 10. The total wall pressure distribution from inlet to the outlet too was recorded. The results indicate that the micro-jets can oblige as the effective regulators of the pressure in the base area. The duct wall pressure field is not negatively affected by the dynamic control. Nozzles were operated with the NPR in the range from 3 to 11. The results show that we can fix the flow parameter which will result in the maximum gain in the base pressure, velocity and temperature. The convergent-divergent nozzle geometry has been modeled and simulated employing turbulence models: K- $\varepsilon$ standard wall function turbulence model from the code was validated with the commercial computational fluid dynamics.
\end{abstract}

Keywords: CFD; C-D Nozzle; ANSYS, Pressure; Mach Number.

\section{Introduction}

An un-expected enlargement of the flow in supersonic regimes is a foremost problem in numerous fluid applications. In sudden expansion problem is an important field in the various engineering problems role in numerous fluid application. In rocket and jet engine test-cells noticed that systems has been used to simulate the upper atmosphere flow field; a jet discharging yields an effective pressure which is sub atmospheric pressure. Moreover, another application observed in the case IC engine. CFD has got its own advantage to simulate the flow field's plays an important role in current technologies of a design optimization by providing the improvised solutions for a given problem. In this paper, the flow has been modelled and analyzed at supersonic Mach number from CD Nozzle. It nozzle has been designed on the assumption of the air to behave as perfect gas compressible flow.

We have considered the flow in the nozzle as an isentropic one. The effect of nozzle pressure ratio is considered for a Mach number 1.87. Pressure and velocity flow in suddenly expanded duct has been computed by evaluating the counters and plots. Moreover, A designed modelled of CD nozzle which can be determine the configuration of suddenly expanded results produced by Sher Afghan Khan \& Rathakrishnan, 2003 using CFD (ANSYS FLUENT) software has been compared.

\section{Literature review}

In early attempts, Anderson et al., (1968) explained base pressure and hence the noise generated by the sudden enlargement of air in a circular duct. Depending on the area ratio and the nozzle's geometry it was noticed that attached flow of base pressure was having a least value. Khan et al., (2002-2012) experimentally investigated the base pressure control using micro-jets and explained the benefits of the flow regulation to control base pressure in a suddenly expanded CD nozzle considering the variables such as the Mach number, area ratio, NPR, and L/D ratio for the cases when control present or absent for the computation of the pressures. However, it is seen that very limited research has been conducted out in the literature to regulate the flow at the base area from a CD nozzle using micro-jets as the control mechanism.

The experimental and computational studies were carried out by various scholars have evaluated the accuracy of the turbulence model for the flow field and the flow parameters of the flow from the nozzle and its performance precisely. Twin methods of Fluidic Thrust Modulation (FTM) were investigated by Ali et al., (2010) (a) Shock Thrust Modulation (STM) and (b) Throat Shifting Thrust Modulation (TSTM). For STM method, the thrust due to the pressure was in the dictating position when modulating the thrust. For TSTM case, the effect of groove dimension to neck size of the nozzle and the interaction of the variables with NPR and the injection direction has significant impacts on the nozzle performance. Su, (2015) investigated the flow-field at the intake of the supersonic ejector using CFD package FLUENT 14.5 along with the ICEM 14.5 to numerically model and test the accomplishment of the ejector performance by two different turbulence models: $\mathrm{k}-\varepsilon$ realizable and $\mathrm{k}-\omega$ shear stress transport (SST) and to enhance the mixing in the ejector's chamber, the wall shape to attain the maximum performance that can be achieved by the ejector. After completing the simulation by the Ansys various contours like pressure, temperature and the pressure ratio plots were obtained. From FLUENT to study the effects of various ejector running variables and also to verify 
the turbulence model accuracy by relating the simulation results with experimental data. Velocity contours, pressure and entrainment ratio plots obtained from FLUENT to investigate the effects of several ejector operating conditions as well as to verify the turbulence model authenticity by relating the simulation results with experimental data. Numerical analysis of flows created by CD nozzle for Mach 2.6 at the nozzle exit to compare available experimental data, obtained during supersonic wind tunnel tests at VTI Zarkovo institute, where major focus was to analyze the performance of flow accelerating device with respect thrust developed and it direction using standard air as the working fluid on a static bed by providing the visible obstructions. Main Focus was on free jet flow, and flow with some obstruction. RANS equations with k$\omega$ SST turbulent model was utilized with structural mesh performance for both cases (Kostic et al., 2015). Exhaustive flow simulation of the flow in a supersonic CD nozzle was studied by Shariatzadeh et al., (2015) using ANSYS FLUENT. The numerical analysis was done in two and three dimension to have a comparison and the effect the three dimensional effect. In the study the performance of various turbulence models was also taken in to account to account on the grid optimization. In the numerical analysis flow variables were optimized for two values of the divergence angles (100 and 50) were considered by Rao \& Akhil, (2016), as the flow variables are not dependent on the diameter of the nozzle even though it affects the flow.

The numerical computations were done for the angle of divergence in the case of CD nozzle at the same time the boundary conditions were unchanged. The analysis has been performed according to the diverged angle of the CD nozzle and keeping the same input conditions and investigated theoretically about sonic, sub-sonic and super-sonic and flow parameters and compared with CFD results. Sahana et al., (2016) analyses the box wing using CFD technique with different flow regimes. Recently, Pathan et al., (2017a, 2017b) numerically investigated the effect of Mach number, area ratio NPR on velocity for an suddenly expanded CD nozzle using ANSYS FLUENT in terms of CFD simulation. Khan et al., (2018) investigated the effect of micro-jets control in suddenly expanded CD nozzle by determining the pressure and velocity effect for different NPR.

\section{Problem definition}

The parameters of the CD nozzle is modelled based on the designed Mach number 1.7 (Fig. 1). Micro-jets are designed $1 \mathrm{~mm}$ of diameter and located at the pitch distance of $1.3 \mathrm{~mm}$ from the divergent diameter. The main aim of this study is to analyze the flows past a $\mathrm{CD}$ nozzle and the computation of the flow parameters such as pressure, and velocity with the effect of different parameters proving by CFD simulation in 2D modeling with and without micro-jets.

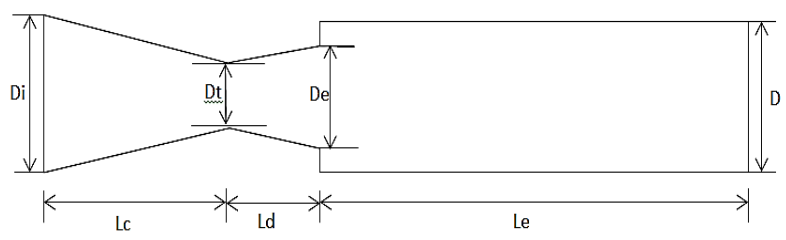

Fig. 1: CD Nozzle with Enlarged Duct.

The dimensions of CD nozzle with suddenly expanded duct are mentioned in table 1 .

Table 1: Dimension of CD Nozzle

\begin{tabular}{ll}
\hline Mach Number & 1.87 \\
\hline Inlet diameter (Di) & $28.72 \mathrm{~mm}$ \\
Throat diameter (Dt) & $8.648 \mathrm{~mm}$ \\
Exit diameter (De) & $10 \mathrm{~mm}$ \\
Extended diameter (D) & $18 \mathrm{~mm}$ \\
Convergent length (Lc) & $35 \mathrm{~mm}$ \\
Divergent length (Ld) & $12.926 \mathrm{~mm}$ \\
Extended length (Le) & $180 \mathrm{~mm}$ \\
Micro-jets diameter (Dm) & $1 \mathrm{~mm}$ \\
\hline
\end{tabular}

\section{Finite element modelling}

The finite element modelling, the fluid flow analyses on CD nozzle observed using ANSYS FLUENT software. ANSYS is a commercial finite element software used in industry to solve a different types of problems. It can be solved fluid flows, structural and thermal problem.

\subsection{Two-dimensional CD nozzle}

For two-dimensional model analyses ANSYS workbench is implemented to model the CD nozzle in case of 2-D planar body. Boundary conditions defined considering edges of the planar body to initiate the solution with perfect ideal gas flow. The 2D planar model of CD nozzle is depicted in Fig. 2(a) and closed form of finite element meshing is depicted in Fig. 2(b). For meshing, ANSYS workbench used and created structural mesh, number of elements has used very high to create fine mesh in closed area at edge of the planar body. Total, 17,032 binary nodes, were generated for 2D planar model.

(A)

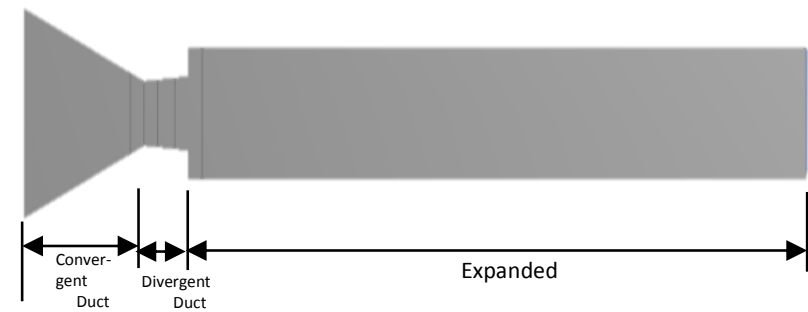

(B)

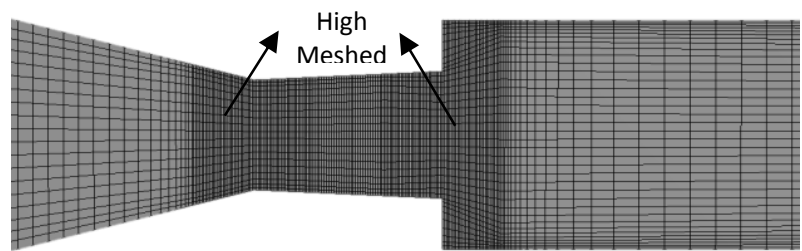

Fig. 2: 2D Planar Fluid Body (A) Finite Element Model (B) Meshing.

\subsection{Calculation procedure}

Computations of the flow field inside the control volume were done using RANS equations with $\mathrm{k}-\varepsilon$ standard turbulent model (ANSYS Inc, 2016, 2017). The most important settings that have been applied are; steady, absolute, 2D planar pressure-based solver. Fluid considered has air with the assumption of air to behave as perfect gas, and for viscosity the Sutherland law was adopted. Boundary conditions were applied inlet is pressure inlet (pa); outlet is pressure outlet. Solution method used in this case is standard pressure; density, momentum, turbulence kinetic energy, turbulence dissipation rate and energy (second order upwind). Finally, solution initialization is considered standard from inlet and the solution run until it converged.

\subsection{Validation of finite element model}

As a first step before going for the final solution; the finite element results, the CD nozzle with micro-jets control and duct located on the divergent portion of the CD nozzle are to be validated and the this is shown in figure 1 of Khan et al., (2002) is considered. By comparing the results obtained by Khan et al., (2003) and the present finite element results, the agreement as presented in table 1. 


\section{Results and discussion}

The aim of research to identify the fluid flow on CD nozzle with and without micro-jets control for a suddenly expanded duct by effect of expansion level. Its effect on pressure, velocity and temperature plots for a designed Mach number 1.87. The problem definition as stated in the Fig. 1 is simplified by observing the fluid flows for each case and identified the effect nozzle pressure ratio (NPR). To obtain this, numerical study was performed and verified each The advantage of the present method is to investigate the perfect fluid flow and active control of pressure micro-jets at the base.

\subsection{Influence of NPR on pressure}

The pressure at the base of the body is key object to study in the fluid mechanics as Bernoulli's principle stated that "when velocity increases pressure will decrease" the same impression is defined in this paper. The Fig. 3 presents the pressure under the stagnation condition and its variation from the inlet to the outlet of the CD nozzle by considering contours and XY plot without control of micro-jets. Similarly, the figure 4 depicts the net pressure variation from the inlet to the outlet of the CD nozzle by considering contours and XY plot with control of micro-jets. Consequently, XY plot as depicted in figure 5 pressure losses when it varies through the wall of the nozzle in both cases, but variation is different for each. The table 2 illustrate the values of total pressure for its influence on NPR with and without micro-jets control.

Also, plotted the static, total and base pressure $(\mathrm{Pb})$ for each NPR shown in figure 6 . The magnitude of pressure at the base area is considered from the abrupt duct diameter at the divergent of the nozzle. In figure 3 and 4 depicts blue color at the expanded duct immediate after divergent portion which represents base pressure that suddenly decreases and emerging rotational flow. The magnitude of static pressure in the re-circulation region is divided into atmospheric pressure $(\mathrm{Pa}) 98273$ Pascal's based on Sher Afghan Khan \& Rathakrishnan (2003) and figure 6 (b) illustrated the base pressure versus wall position. Finally, base pressure versus NPR also represented with and without micro-jets as depicted in figure 7. The outcome base on CFD show that the loss of pressure is minimum when micro-jets installed at the base and flow of rotation also become control.
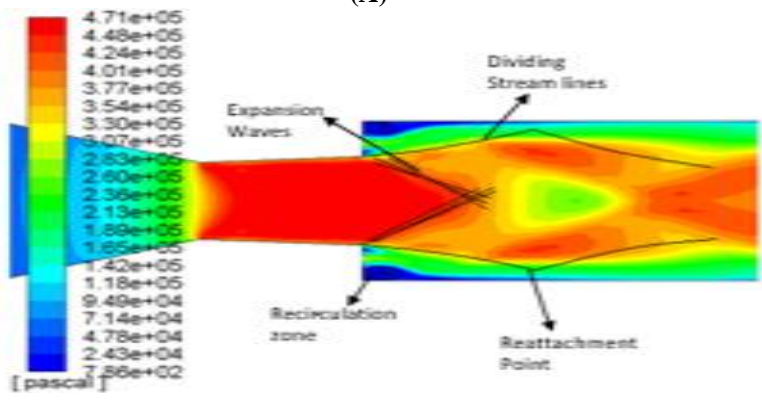

(B)

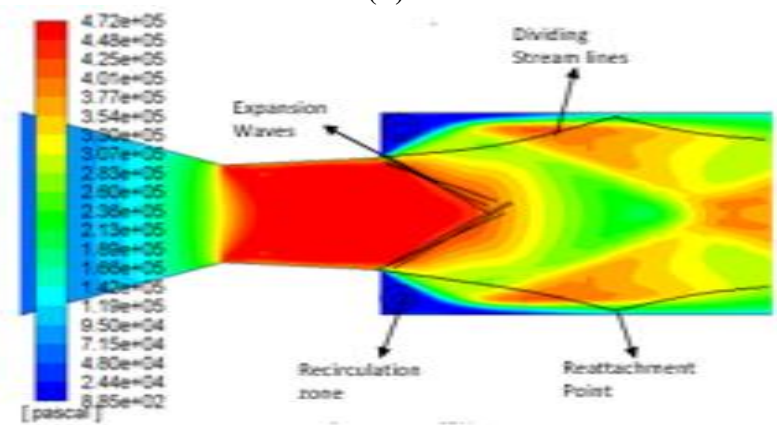

Fig. 3: Close View of CD Nozzle with Pressure Flow (A) with Control (B) Without Control.

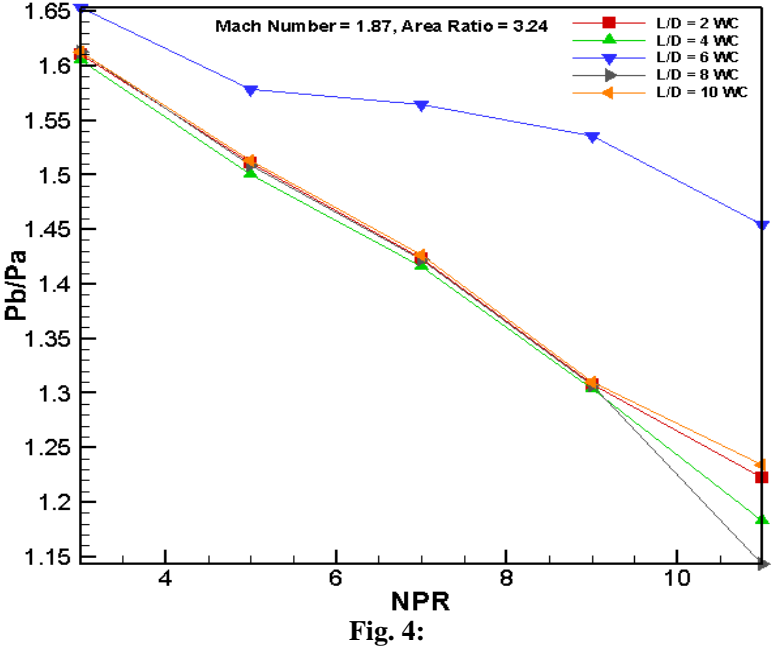

\subsection{NPR Effect on velocity (Mach number)}

The velocity is considering by Mach number in a fluid body is also important. The figure 8 and 9 shows the Mach number variation from the inlet to the outlet of the CD nozzle by considering contours and XY plot without as well as with control of micro-jets. Consequently, XY plot shown in Fig. 10 Mach number increases when it varies through the wall of the nozzle. The table 3 illustrate the values of Mach number for an effect of nozzle pressure ration with and without micro-jets control.

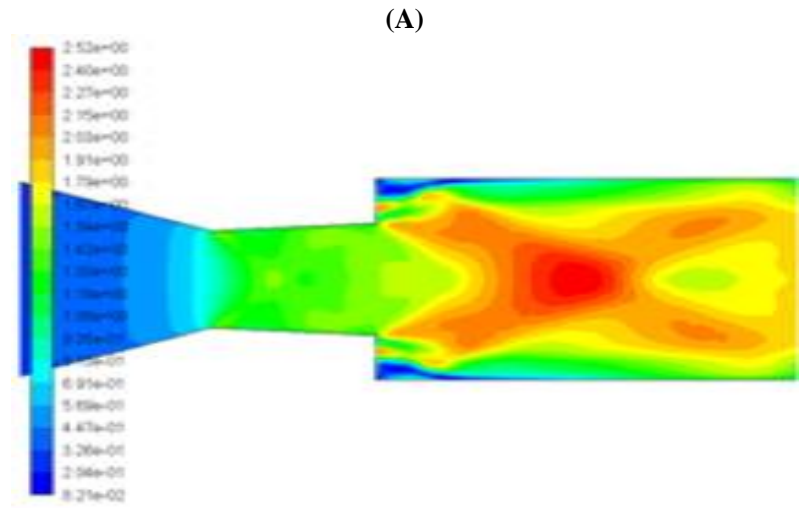

(B)

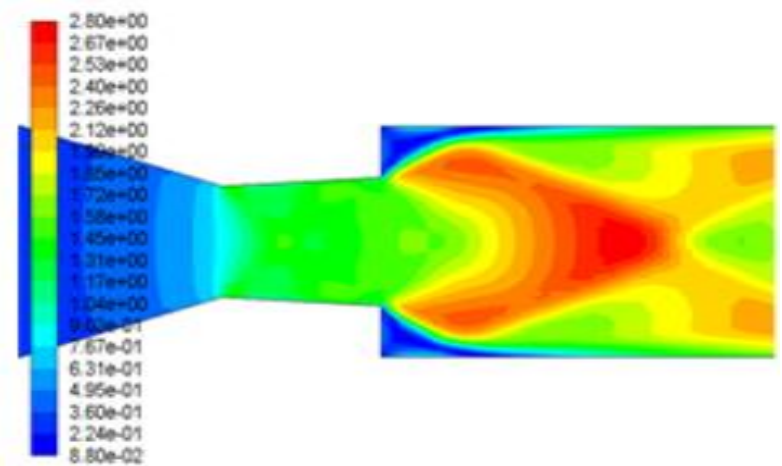

Fig. 3: Close View of CD Nozzle with Velocity (Mach number) (A) with Control (B) Without Control.

In Fig. 4(a) indicates that control is not effective due to this the pressure suddenly decreases and creating shocks at the exit of the divergent and at the base flow of pressure become high therefore the shocks may have chance to increase drag at the expanded duct. To control this the small diameter of micro-jets are used shown in figure 11(b). The effect of micro-jets are proved at the base the rotation flow of air decreased.

Furthermore, to regulate the pressure at base Khan et al., (20022012) experimentally investigated with and without control of fluid 
flows in a CD nozzle with effect of NPR, area ratio and L/D of expanded nozzle. Similarly, for NPR 5, 7, 9 and 11 the velocity vector results approximately same rotational flows at the base of expanded duct only it slightly differs in area as compared to NPR 3 .

\section{Conclusion}

The effect of the NPR on pressure, velocity and temperature for suddenly expanded convergent-divergent nozzle and the dynamic control in the form of tiny-jets to regulate the pressure at base has been demonstrated using ANSYS workbench 18.0. The base pressure assumes ratio area 6.25 and $\mathrm{L} / \mathrm{D}=10$ at 1.87 Mach number. The micro-jets are found to be effective for a fixed level of inertia and the expansion level. The static pressure in the duct is not negatively affected by the active control.

With the discussion we conclude that, total pressure dramatically varying from inlet to the outlet and value of pressure is high for NPR 11 as shown in table 2. As pressure decreases velocity will increase and our results proved that the velocity is high at the exit and variation of pressure inlet to the outlet is observed by considering Mach number shown in table 3. For satisfactory results also identified the efficacy of level of expansion on temperature for suddenly expanded flows. Slightly decreases temperature from inlet to the outlet for static temperature shown in table 4. Finally, 2D mesh model is depicted in Fig. 2. Mesh generated for 2D is 21,368 binary nodes structural mesh has been used.

\section{References}

[1] Ahmed, M., \& Baig, A. L. I. (2012). Active control of base pressure in suddenly expanded flow for area ratio 4. 84. International Journal of Engineering Science and Technology, 4(5), 1892-1902.

[2] Ali, A., Neely, A., Young, J., Blake, B., \& Lim, J. Y. (2010). Numerical Simulation of Fluidic Modulation of Nozzle Thrust. In 17th Australasian Fluid Mechanics Conference (pp. 5-8).

[3] Anderson, J. S., \& Williams, T. J. (1968). Base pressure and noise produced by the abrupt expansion of air in a cylindrical duct. Journal of Mechanical Engineering Science, 10(3), 262-268.

[4] ANSYS Inc. (2016). ANSYS FLUENT 15.0: Theory Guidance. Canonsburg PA.

[5] ANSYS Inc. (2017). ANSYS FLUENT 18.0: Theory Guidance. Canonsburg PA.

[6] Khan, S. A., \& Aabid, A. (2018). CFD Analysis of CD Nozzle and Effect of Nozzle Pressure Ratio on Pressure and Velocity for Suddenly Expanded Flows. International Journal of Mechanical and Production Engineering Research and Development (IJMPERD), 8(June), 1147-1158.

[7] Khan, S. A., \& Rathakrishnan, E. (2002). Active Control of Suddenly Expanded Flows from Overexpanded Nozzles. International Journal of Turbo and Jet Engines, 19, 119-126.

[8] Khan, S. A., \& Rathakrishnan, E. (2003). Control of Suddenly Expanded Flows with Micro-Jets. International Journal of Turbo and Jet Engines, 20, 63-82.

[9] Khan, S. A., \& Rathakrishnan, E. (2004a). Active Control of Suddenly Expanded Flows from Underexpanded Nozzles. International Journal of Turbo and Jet Engines, 21, 233-254.

[10] Khan, S. A., \& Rathakrishnan, E. (2004b). Control of Suddenly Expanded Flows from Correctly Expanded Nozzles. International Journal of Turbo and Jet Engines, 21, 255-278.

[11] Khan, S. A., \& Rathakrishnan, E. (2005). Active Control of Suddenly Expanded Flows from Underexpanded Nozzles - Part II. International Journal of Turbo and Jet Engines, 22, 163-183.

[12] Khan, S. A., \& Rathakrishnan, E. (2006). Control of suddenly expanded flow. Aircraft Engineering and Aerospace Technology: An International Journal, 78(4), 293-309. https://doi.org/10.1108/17488840610675573.

[13] Khan, S. A., \& Rathakrishnan, E. (2006). Nozzle Expansion Level Effect on Suddenly Expanded Flow Sher. International Journal of Turbo and Jet Engines, 23, 233-257.

[14] Kostic, O., Stefanovic, Z., \& Kostic, I. (2015). CFD modeling of supersonic airflow generated by $2 \mathrm{D}$ nozzle with and without an obstacle at the exit section. FME Transaction, 43(2), 107-113. https://doi.org/10.5937/fmet1502107k.
[15] Pathan, K. A., Khan, S. A., \& Dabeer, P. S. (2017a). CFD Analysis of Effect of Area Ratio on Suddenly Expanded Flows. In second International Conference for Convergence in Technology (I2CT) CFD (pp. 1192-1198)

[16] Pathan, K. A., Khan, S. A., \& Dabeer, P. S. (2017b). CFD Analysis of Effect of Flow and Geometry Parameters on Thrust Force Created by Flow from Nozzle. In second International Conference for Convergence in Technology (I2CT) CFD (pp. 1121-1125).

[17] Pathan, K. A., Khan, S. A., \& Dabeer, P. S. (2017c). CFD Analysis of Effect of Mach number, Area Ratio and Nozzle Pressure Ratio on Velocity for Suddenly Expanded Flows. In second International Conference for Convergence in Technology (I2CT) CFD (pp. 11041110).

[18] Rao, A. V. R., \& Akhil, I. (2016). CFD analysis of CD nozzle at two divergent angles for prediction of Mach number $(M<1, M=1 \&$ $\mathrm{M}>1$ ) and flow parameters of newtonian fluid by using ANSYSFLUINT 14.0. International Journal of Engineering Sciences and Research Technology, 5(5), 413-428.

[19] Rehman, S., \& Khan, S. A. (2008). Control of base pressure with micro-jets : part I. Aircraft Engineering and Aerospace Technology, 80(2), 158-164. https:// doi.org/ 10.1108 /000226 60810859373.

[20] Sahana, D. S., \& Aabid, A. (2016). CFD Analysis of Box Wing Configuration. International Journal of Science and Research (IJSR), 5(4), 706-709.

[21] Shariatzadeh, O. J., Abrishamkar, A., \& Jafari, A. J. (2015). Computational Modeling of a Typical Supersonic Converging-Diverging Nozzle and Validation by Real Measured Data. Journal of Clean Energy Technologies, 3(3), 220-225. https://doi.org/10.7763/JOCET.2015.V3.198.

[22] Su, L. (2015). CFD Simulation and Shape Optimization of Supersonic Ejectors for Refrigeration and. 